\title{
Global existence and boundedness in a reaction-diffusion-taxis system with three species
}

Xue $\mathrm{Xu}^{1,2^{*}}$ (1) and Yong Wang ${ }^{1}$

\section{"Correspondence:}

xuxue06@163.com

'Department of Mathematics,

Harbin Institute of Technology,

Harbin, P.R. China

${ }^{2}$ Department of Mathematics,

Harbin University, Harbin, P.R. China

\begin{abstract}
The global existence and boundedness of a reaction-diffusion-taxis system with three interacting species, among which two species consist of predators competing for one species of prey, are investigated.
\end{abstract}

MSC: 35K57; 35K59; 35B45; 92D25

Keywords: Reaction-diffusion-taxis; Predator-prey; Global existence; Boundedness

\section{Introduction}

The predator-prey model [1-6] is a basic differential equation model describing the interaction among species, and considerable work has been done with regards to both Turing and non-Turing patterns where the latter often appears to be chaotic space [7, 8]. In general, the coexistence of prey population and predator population can be mostly described by the presence of positive steady states, while spatial patterns of the populations can be characterized by non-constant steady states, which have been studied for population models with random diffusions by various types of mathematical models, e.g. [9-11].

In reality, prey are pursued and escape from predators in the spatial movement. Such a movement is not random but directed. The object of this paper is to study the problem for three interacting species, among which two species of predators compete for one species of prey. The model is taken to be

$$
\begin{cases}\frac{\partial u}{\partial t}=d_{1} \Delta u-f_{1}(u) v_{1}-f_{2}(u) v_{2}, & x \in \Omega, t>0, \\ \frac{\partial v_{1}}{\partial t}=d_{2} \Delta v_{1}-\chi_{1} \nabla\left(S\left(v_{1}\right) \nabla u\right)+v_{1}\left[f_{1}(u)-v_{1}-v_{2}\right], & x \in \Omega, t>0, \\ \frac{\partial v_{2}}{\partial t}=d_{3} \Delta v_{2}-\chi_{2} \nabla\left(S\left(v_{2}\right) \nabla u\right)+v_{2}\left[f_{2}(u)-v_{1}-v_{2}\right], & x \in \Omega, t>0, \\ \frac{\partial u}{\partial n}=\frac{\partial v_{1}}{\partial n}=\frac{\partial v_{2}}{\partial n}=0, & x \in \partial \Omega, t>0, \\ u(x, 0)=u_{0}(x) \geq 0, \quad v_{1}(x, 0)=v_{10}(x) \geq 0, & \\ v_{2}(x, 0)=v_{20}(x) \geq 0, & x \in \Omega,\end{cases}
$$

where $v_{1}, v_{2}$ and $u$ are the population densities of two predator species and one prey species, respectively; $\Omega$ is a bounded domain in $\mathbb{R}^{N}(N \geq 1)$ with a smooth boundary $\partial \Omega$;

(c) The Author(s) 2018. This article is distributed under the terms of the Creative Commons Attribution 4.0 International License (http://creativecommons.org/licenses/by/4.0/), which permits unrestricted use, distribution, and reproduction in any medium, provided you give appropriate credit to the original author(s) and the source, provide a link to the Creative Commons license, and indicate if changes were made. 
$n$ is the unit outer normal, and no flux boundary condition is imposed so the system is a closed one. Here $f_{i}(u)$ satisfies:

$(F) f_{i}(u)$ is continuously differentiable from $\mathbb{R}_{+} \rightarrow \mathbb{R}_{+}$and $f_{i}(0)=0, f_{i}^{\prime}(u) \geq 0, i=1,2$.

Biologically, $f_{i}(u)$ represents the consumption rate of the prey per predator. A typical example of $f_{i}$ is the monotone Holling Type II function, $f_{i}(u)=m_{i} u /\left(a_{i}+u\right)$, where $m_{i}>0$ is the maximal growth rate and $a_{i}>0$ is the half-saturation constant. The forms of $F_{1}=v_{1}\left[f_{1}(u)-v_{1}-v_{2}\right]$ and $F_{2}=v_{2}\left[f_{2}(u)-v_{1}-v_{2}\right]$ represent the constant level of the prey $u$, and the predators $v_{1}, v_{2}$ have logistic growth. It is assumed that the predators $v_{1}, v_{2}$ are attracted by the preys $u$, so they move in the direction proportional to the negative gradient of prey population. That is modeled by a prey-taxis term $\chi_{i} \nabla\left(S\left(v_{i}\right) \nabla u\right), i=1,2$, respectively, where the $\chi_{i}$ are the prey-taxis coefficients, and the movement is decided also by the predator's density, which is indicated by the function $S\left(v_{i}\right)$. As pointed in [12], the sensitivity function $S(u)$ satisfies the general hypotheses:

$\left(H_{1}\right) S:[0, \infty) \rightarrow[0, \infty)$ is continuously differentiable and $S(0)=0$;

$\left(H_{2}\right)$ There exists $C>0$ such that $S(u) \leq C u$ for any $u \geq 0$ and $x \in \bar{\Omega}$.

For example, in [13], the sensitivity function $q(u)$ can take the form

$$
S(u)=u, \quad S(u)=\frac{u}{1+\varepsilon u}, \quad S(u)=u e^{-\varepsilon u},
$$

where $\varepsilon>0, m \geq 1$.

This paper concentrates on the global existence and boundedness of system (1.1).

\section{Local existence and preliminaries}

It is noticed that (1.1) has a unique non-negative local-in-time classical solution $(u(x, t)$, $\left.v_{1}(x, t), v_{2}(x, t)\right)$ by using the abstract theory of quasilinear parabolic systems in [14]. Moreover, we can obtain the following results.

Lemma 2.1 Assume that $\left(H_{1}\right),\left(H_{2}\right),(F)$ hold and the initial data $u_{0} \in W^{1, p}(\Omega)$ for $p>n$ and $v_{i 0} \in\left(W^{1, p}(\Omega)\right)^{2}$ for $p>n(i=1,2)$. Then

1. There exists a positive constant $T_{\max }$ (the maximal existence time) such that the system (1.1) has a unique non-negative classical solution $\left(u(x, t), v_{1}(x, t), v_{2}(x, t)\right)$ satisfying $\left(u(x, t), v_{1}(x, t), v_{2}(x, t)\right) \in\left(C\left(\left[0, T_{\max }\right) ; W^{1, p}(\Omega)\right) \cap C^{2,1}\left(\bar{\Omega} \times\left(0, T_{\max }\right)\right)\right)^{3}$ and

$$
v_{1}(x, t) \geq 0, \quad v_{2}(x, t) \geq 0, \quad u_{*} \geq u(x, t) \geq 0, \quad x \in \Omega, 0 \leq t<T_{\max },
$$

where $u_{*}>0$ is a constant satisfying $\int_{\Omega} u_{0}(x)=|\Omega| u_{*}$.

2. Iffor each $T>0$, there exists a constant $M_{0}(T)$ such that

$$
\left\|\left(u(x, t), v_{1}(x, t), v_{2}(x, t)\right)\right\|_{\infty} \leq M_{0}(T), \quad 0<t<\min \left\{T, T_{\max }\right\}
$$

where $M_{0}(T)$ is a constant depending on $T$ and $\left\|\left(u_{0}, v_{10}, v_{20}\right)\right\|_{1, p}$, then $T_{\max }=+\infty$.

Proof From Theorem 14.6 in [14], the local existence of $\left(u(x, t), v_{1}(x, t), v_{2}(x, t)\right)$ is obtained. It is noticed that every equation in (1.1) can be treated as a scalar linear equation in $u$ and $v_{1}, v_{2}$, so we have $u(x, t) \geq 0, v_{i}(x, t) \geq 0(i=1,2)$ from $u_{0} \geq 0, v_{i 0} \geq 0$ and the maximum principle for parabolic equation. 
Also from (1.1) and $v_{1}(x, t) \geq 0, v_{2}(x, t) \geq 0$, we have

$$
\begin{cases}\frac{\partial u}{\partial t}=d_{3} \Delta u-f_{1}(u) v_{1}-f_{2}(u) v_{2} \leq d_{3} \Delta u, & x \in \Omega, t>0, \\ \frac{\partial u}{\partial v}=0, & x \in \partial \Omega, \\ u(x, 0)=u_{0}(x) \geq 0, & x \in \Omega .\end{cases}
$$

Using the Neumann boundary condition and the comparison principle again, we obtain $u \leq u_{*}$, where $u_{*}>0$ is a constant satisfying $\int_{\Omega} u_{0}(x)=|\Omega| u_{*}$. Part 2 follows from Theorem 15.5 in [15], so we have $T_{\max }=\infty$.

In order to obtain the global existence of non-negative solutions for system (1.1), we recall some preliminary estimates which will be used in our proof. First we review some well-known estimates for the diffusion semigroup with homogeneous Neumann boundary conditions (see [16]). For $p \in(1, \infty)$, let $A$ denote the sectorial operator defined by

$$
A u:=-\Delta u \quad \text { for } u \in D(A):=\left\{\omega \in W^{2, p}(\Omega): \frac{\partial \omega}{\partial n}=0 \text { on } \partial \Omega\right\} .
$$

Similarly we let $A_{d} u=-d \Delta u$ which satisfies the same properties as $A$ with a scaling. Then we only collect properties of $A$ here while the same properties for $A_{d}$ will be applied in the following analysis.

Lemma 2.2 Assume that $m \in\{0,1\}, p \in[1, \infty]$ and $q \in(1, \infty)$. Then there exists some positive constant $C_{2}$, such that

$$
\|u\|_{m, p} \leq C_{2}\left\|(A+1)^{\theta} u\right\|_{q}
$$

for any $u \in D\left((A+1)^{\theta}\right)$, where $\theta \in(0,1)$ satisfies

$$
m-\frac{n}{p}<2 \theta-\frac{n}{q}
$$

If in addition $q \geq p$, then there exist $C_{3}>0$ and $\gamma>0$ such that, for any $u \in L^{p}(\Omega)$,

$$
\left\|(A+1)^{\theta} e^{-t(A+1)} u\right\|_{q} \leq C_{3} t^{-\theta-\frac{n}{2}\left(\frac{1}{p}-\frac{1}{q}\right)} e^{-\gamma t}\|u\|_{p}
$$

where the associated diffusion semigroup $\left\{e^{-t(A+1)}\right\}_{t \geq 0}$ maps $L^{p}(\Omega)$ into $D\left((A+1)^{\theta}\right)$. Moreover, for any $p \in(1, \infty)$ and $\varepsilon>0$, there exist $C_{4}>0$ and $\mu>0$ such that

$$
\left\|(A+1)^{\theta} e^{-t A} \nabla \cdot u\right\|_{p} \leq C_{4} t^{-\theta-\frac{1}{2}-\varepsilon} e^{-\mu t}\|u\|_{p}
$$

which is valid for all $\mathbb{R}^{n}$-valued $u \in L^{p}(\Omega)$.

\section{Global existence and boundedness of solutions}

In this section we prove the global existence and boundedness of solutions for system (1.1). The main step toward the result is to establish a uniform bound of $v_{1}(x, t), v_{2}(x, t)$ in $L^{k}(\Omega)$ for any $k \geq 2$. First we show that the solution $v_{1}(x, t)$ and $v_{2}(x, t)$ are bounded in $L^{1}(\Omega)$. 
Lemma 3.1 Assume that $\left(H_{1}\right),\left(H_{2}\right)$ and $(F)$ hold. Then there exists a constant $C_{0}>0$ such that the predator component of (1.1) satisfies the following estimate:

$$
\int_{\Omega} v_{1}(x, t)+v_{2}(x, t) d x<C_{0} \quad \text { for all } t \in\left(0, T_{\max }\right) .
$$

Proof Let $\int_{\Omega} v_{i}(x, t) d x=Q_{i}(t)(i=1,2), \int_{\Omega} u(x, t) d x=Q_{3}(t)$. Then we have

$$
\begin{aligned}
\frac{\partial Q_{1}}{\partial t} & =\int_{\Omega} d_{2} \Delta v_{1}-\chi_{1} \nabla\left(S\left(v_{1}\right) \nabla u\right)+v_{1}\left(f_{1}(u)-v_{1}-v_{2}\right) d x \\
& =d_{2} \int_{\Omega} \frac{\partial v_{1}}{\partial v} d S-\chi_{1} \int_{\Omega} S\left(v_{1}\right) \frac{\partial u}{\partial v} d S+\int_{\Omega} v_{1}\left(f_{1}(u)-v_{1}-v_{2}\right) d x, \\
\frac{\partial Q_{2}}{\partial t} & =\int_{\Omega} d_{3} \Delta v_{2}-\chi_{2} \nabla\left(S\left(v_{2}\right) \nabla u\right)+v_{2}\left(f_{2}(u)-v_{1}-v_{2}\right) d x \\
& =d_{3} \int_{\Omega} \frac{\partial v_{2}}{\partial v} d S-\chi_{2} \int_{\Omega} S\left(v_{2}\right) \frac{\partial u}{\partial v} d S+\int_{\Omega} v_{2}\left(f_{2}(u)-v_{1}-v_{2}\right) d x, \\
\frac{\partial Q_{3}}{\partial t} & =\int_{\Omega} d_{1} \Delta u-f_{1}(u) v_{1}-f_{2}(u) v_{2} d x \\
& =d_{1} \int_{\Omega} \frac{\partial u}{\partial v} d S+\int_{\Omega}-f_{1}(u) v_{1}-f_{2}(u) v_{2} d x .
\end{aligned}
$$

From the Neumann boundary conditions and the uniform boundedness of $u$ in Lemma 2.1, we have

$$
\frac{\partial\left(Q_{1}+Q_{2}+Q_{3}\right)}{\partial t}=\int_{\Omega}-\left(v_{1}+v_{2}\right)^{2} d x \leq 0
$$

Consequently,

$$
Q_{1}+Q_{2}<Q_{1}+Q_{2}+Q_{3} \leq \int_{\Omega}\left(u_{0}+v_{10}+v_{20}\right) d x:=C_{0} .
$$

Now we carry out the $L^{p}$ bound of $v_{1}, v_{2}$ for $p \geq 2$. The following Gagliardo-Nirenberg inequality plays a key role in our proof (see [17] for detail).

Lemma 3.2 Let $u \in L^{p}(\Omega)$ and $D^{k} u \in L^{q}(\Omega)$ where $p, q \in[1, \infty]$. Then, for the derivatives $D^{i} u, i \in[0, k)$, there exists a constant $C_{5}>0$ such that

$$
\left\|D^{i} u\right\|_{h} \leq C_{5}\left(\left\|D^{k} u\right\|_{q}^{\lambda}\|u\|_{p}^{1-\lambda}+\|u\|_{m}\right)
$$

where

$$
\frac{1}{h}-\frac{i}{n}=\lambda\left(\frac{1}{q}-\frac{k}{n}\right)+(1-\lambda) \frac{1}{p}, \quad m>0,
$$

and $\lambda$ satisfies

$$
\frac{i}{k} \leq \lambda \leq 1 \text {. }
$$

Moreover, we recall the following elementary inequality (see [18]). 
Lemma 3.3 Assume that $y, z \in \mathbb{R}, y, z \geq 0$ and $r>0$, then we have

$$
(y+z)^{r} \leq 2^{r}\left(y^{r}+z^{r}\right)
$$

Theorem 3.4 Assume that $(F),\left(H_{1}\right)$ and $\left(H_{2}\right)$ hold. Then, for any $k \geq 2$, there exists a positive constant $C_{1}>0$ such that

$$
\left\|v_{1}(\cdot, t)\right\|_{k} \leq C_{1}, \quad\left\|v_{2}(\cdot, t)\right\|_{k} \leq C_{1}, \quad \text { for all } t \in\left(0, T_{\max }\right)
$$

Proof First we show that, for any $\tau \in\left(0, T_{\max }\right)$, there exists a constant $H(\tau)>0$ such that

$$
\|u(\cdot, t)\|_{1, \infty} \leq H(\tau), \quad \text { for all } t \in\left(\tau, T_{\max }\right) .
$$

Let $\tau \in\left(0, T_{\max }\right)$ be given such that $\tau<1$, and choose $q>n$ and $\theta \in\left(\frac{1}{2}\left(1+\frac{n}{q}\right), 1\right)$. The first equation of (1.1) can be rewritten as

$$
\frac{\partial u}{\partial t}=d_{1} \Delta u-u+\varphi\left(u, v_{1}, v_{2}\right)
$$

where $\varphi\left(u, v_{1}, v_{2}\right)=\left(u-f_{1}(u) v_{1}-f_{2}(u) v_{2}\right)$. Then from the variation of constants formula for (3.8), we have

$$
u(\cdot, t)=e^{-t\left(A_{d_{1}}+1\right)} u_{0}+\int_{0}^{t} e^{-(t-s)\left(A_{d_{1}}+1\right)} \varphi\left(u(\cdot, t), v_{1}(\cdot, t), v_{2}(\cdot, t)\right) d s .
$$

From (2.4) and (2.5) we have

$$
\begin{aligned}
\|u(\cdot, t)\|_{1, \infty} & \leq C_{2}\left\|\left(A_{d_{1}}+1\right)^{\theta} u(\cdot, t)\right\|_{q} \\
& \leq C_{3} \int_{0}^{t}(t-s)^{-\theta} e^{-\gamma(t-s)}\left\|u-f_{1}(u) v_{1}-f_{2}(u) v_{2}\right\|_{q} d s+C_{3} t^{-\theta} e^{-\gamma t}\left\|u_{0}\right\|_{q} \\
& \leq C_{3} \int_{0}^{t}(t-s)^{-\theta} e^{-\gamma(t-s)}\|u(\cdot, t)\|_{\infty} d s+C_{3} t^{-\theta} e^{-\gamma t}\left\|u_{0}\right\|_{q} \\
& \leq C_{3} t^{-\theta}+C_{3} \int_{0}^{t}(t-s)^{-\theta} e^{-\gamma(t-s)} d s \leq C_{3} t^{-\theta}+C_{3} \int_{0}^{\infty} \sigma^{-\theta} e^{-\gamma \sigma} d \sigma \\
& \leq C_{3}\left(\tau^{-\theta}+1\right):=H(\tau) \quad \text { for all } t \in\left(\tau, T_{\max }\right),
\end{aligned}
$$

where $C_{3}$ denotes a generic constant that may vary from line to line. For any $k \geq 2$, from (1.1), (3.9), $\left(H_{2}\right)$ and Young's inequality, we obtain

$$
\begin{aligned}
\frac{d}{d t} \int_{\Omega} v_{1}^{k}= & k \int_{\Omega} v_{1}^{k-1}\left(v_{1}\right)_{t} \\
\leq & k \int_{\Omega} v_{1}^{k-1} d_{2} \Delta v_{1}+k \int_{\Omega} v_{1}^{k-1} \chi_{1} \nabla \cdot\left(S\left(v_{1}\right) \nabla u\right)+k \int_{\Omega} v_{1}^{k-1} v_{1}\left(f_{1}(u)-v_{1}-v_{2}\right) \\
= & -k(k-1) d_{2} \int_{\Omega} v_{1}^{k-2}\left|\nabla v_{1}\right|^{2}-k(k-1) \chi_{1} \int_{\Omega} v_{1}^{k-2} S\left(v_{1}\right) \nabla u \cdot \nabla v_{1} \\
& +k \int_{\Omega} v_{1}^{k-1} v_{1}\left(f_{1}(u)-v_{1}-v_{2}\right)
\end{aligned}
$$




$$
\begin{aligned}
\leq & \frac{-4(k-1) d_{2}}{k} \int_{\Omega}\left|\nabla v_{1}^{\frac{k}{2}}\right|^{2}+I k(k-1) \int_{\Omega} v_{1}^{k-2} S\left(v_{1}\right)\left|\nabla v_{1}\right|+k \int_{\Omega} v_{1}^{k-1} v_{1} f_{1}(u) \\
\leq & \frac{-4(k-1) d_{2}}{k} \int_{\Omega}\left|\nabla v_{1}^{\frac{k}{2}}\right|^{2}+C I k(k-1) \int_{\Omega} u_{1}^{k-1}\left|\nabla v_{1}\right|+E k \int_{\Omega} v_{1}^{k} \\
= & \frac{-4(k-1) d_{2}}{k} \int_{\Omega}\left|\nabla v_{1}^{\frac{k}{2}}\right|^{2}+C I k(k-1) \frac{2}{k} \int_{\Omega} v_{1}^{\frac{k}{2}}\left|\nabla v_{1}^{\frac{k}{2}}\right|+E k \int_{\Omega} v_{1}^{k} \\
\leq & \frac{-4(k-1) d_{2}}{k} \int_{\Omega}\left|\nabla v_{1}^{\frac{k}{2}}\right|^{2} \\
& +C I(k-1)\left(\frac{2}{C I k} \int_{\Omega}\left|\nabla v_{1}^{\frac{k}{2}}\right|^{2}+\frac{C I k}{2} \int_{\Omega} v_{1}^{k}\right)+E k \int_{\Omega} v_{1}^{k} \\
\leq & \frac{-2(k-1) d_{2}}{k} \int_{\Omega}\left|\nabla v_{1}^{\frac{k}{2}}\right|^{2}+\left(\frac{C^{2} I^{2} k(k-1)}{2}+E k\right) \int_{\Omega} v_{1}^{k}
\end{aligned}
$$

where $I=\chi_{1} H(\tau)$ is a positive constant. Then we have

$$
\frac{d}{d t} \int_{\Omega} v_{1}^{k} \leq \frac{-2(k-1) d_{2}}{k} \int_{\Omega}\left|\nabla v_{1}^{\frac{k}{2}}\right|^{2}+\left(\frac{C^{2} I^{2} k(k-1)}{2}+E k c\right) \int_{\Omega} v_{1}^{k}
$$

From Lemma 3.2 and Lemma 3.3, we find that

$$
\begin{aligned}
\int_{\Omega} v_{1}^{k} & =\left\|v_{1}^{\frac{k}{2}}\right\|_{2}^{2} \\
& \leq C_{5}\left(\left\|\nabla v_{1}^{\frac{k}{2}}\right\|_{2}^{\lambda}\left\|v_{1}^{\frac{k}{2}}\right\|_{\frac{2}{k}}^{1-\lambda}+\left\|v_{1}^{\frac{k}{2}}\right\|_{\frac{2}{k}}\right)^{2} \\
& \leq C_{5}\left(\left\|\nabla v_{1}^{\frac{k}{2}}\right\|_{2}^{\lambda}\left\|1+v_{1}\right\|_{1}^{\frac{k}{2}(1-\lambda)}+\left\|1+v_{1}\right\|_{1}^{\frac{k}{2}}\right)^{2} \\
& \leq C_{5}\left(\left\|\nabla v_{1}^{\frac{k}{2}}\right\|_{2}^{\lambda}\left(|\Omega|+C_{1}\right)^{\frac{k}{2}(1-\lambda)}+\left(|\Omega|+C_{1}\right)^{\frac{k}{2}}\right)^{2} \\
& \leq C_{6}\left(\left\|\nabla v_{1}^{\frac{k}{2}}\right\|_{2}^{2 \lambda}+1\right),
\end{aligned}
$$

where

$$
\lambda=\frac{k n-n}{2+k n-n} \in(0,1)
$$

for any $k \geq 2$. Since (3.13) implies that $2 \lambda<2$, then from (3.12) we obtain

$$
\int_{\Omega} v_{1}^{k} \leq C_{7}\left(\left\|\nabla v_{1}^{\frac{k}{2}}\right\|_{2}^{2}+1\right)
$$

By using Young's inequality and (3.14), we obtain

$$
\left(\frac{C^{2} I^{2} k(k-1) d_{1}}{2}+E k c+1\right) \int_{\Omega} v_{1}^{k} \leq \frac{2(k-1) d_{1}}{k} \int_{\Omega}\left|\nabla v_{1}^{\frac{k}{2}}\right|^{2}+C_{8},
$$

for some $C_{8}>0$. Combining (3.11) and (3.15), we have

$$
\frac{d}{d t} \int_{\Omega} v_{1}^{k}+\int_{\Omega} v_{1}^{k} \leq C_{8}
$$


Integrating (3.16), we arrive at

$$
\int_{\Omega} v_{1}^{k} \leq \max \left\{\int_{\Omega} v_{10}^{k}, C_{8}\right\}:=R_{1} .
$$

Similarly, we can get

$$
\int_{\Omega} v_{2}^{k} \leq \max \left\{\int_{\Omega} v_{20}^{k}, C_{9}\right\}:=R_{2},
$$

which are the desired results.

Next we establish the $L^{\infty}$ bound for $v_{1}(x, t), v_{2}(x, t)$. The following Sobolev inequality will be used in forthcoming proofs.

Lemma 3.5 Let

$$
2^{*}= \begin{cases}\infty, & n \leq 2, \\ \frac{n}{n-2}, & n>2 .\end{cases}
$$

Then, for any $1<\alpha \leq 2^{*}$ and $k>0$, there exists a positive constant $M_{0}$ such that

$$
\left(\int_{\Omega} u^{(k+1) \alpha} d x\right)^{\frac{1}{\alpha}} \leq M_{0} \int_{\Omega}\left(\left|\nabla\left(u^{\frac{k+1}{2}}\right)\right|^{2}+u^{k+1}\right) d x
$$

We make a key progress on the boundedness estimates of $v_{1}, v_{2}$.

Theorem 3.6 Let $\left(u(x, t), v_{1}(x, t), v_{2}(x, t)\right)$ be the solution of (1.1). Assume that $(F),\left(H_{1}\right)$ and $\left(\mathrm{H}_{2}\right)$ hold, then there exists a positive constant $M$ such that

$$
\left\|v_{1}(\cdot, t)\right\|_{\infty} \leq M, \quad\left\|v_{2}(\cdot, t)\right\|_{\infty} \leq M \quad \text { for all } t \in\left(0, T_{\max }\right) .
$$

Proof We use semigroup arguments $($ see $[13,16])$ to get the $L^{\infty}$-bound of $v_{1}, v_{2}$. As pointed out in Theorem 3.4, by using the variation of constants formula, we have

$$
\begin{aligned}
v_{1}(\cdot, t)= & e^{-t\left(A_{d_{2}}+1\right)} v_{10}-\chi_{1} \int_{0}^{t} e^{-(t-s)\left(A_{d_{2}}+1\right)} \nabla \cdot\left(S\left(v_{1}(\cdot, t)\right) \nabla u(\cdot, t)\right) d s \\
& +\int_{0}^{t} e^{-(t-s)\left(A_{d_{2}}+1\right)} \psi\left(v_{1}(\cdot, t), v_{2}(\cdot, t), u(\cdot, t)\right) d s \\
:= & E_{1}+E_{2}+E_{3},
\end{aligned}
$$

where $\psi\left(u(\cdot, t), v_{1}(\cdot, t), v_{2}(\cdot, t)\right)=v_{1}\left(f_{1}(u)-v_{1}-v_{2}\right)$. Then we estimate the $L^{\infty}$-bound for each of $E_{1}, E_{2}$ and $E_{3}$ separately. We also choose $\tau<1$ as done in Theorem 3.4.

For $E_{1}$, we find that

$$
\left\|E_{1}(\cdot, t)\right\|_{\infty} \leq C_{3} \tau^{\vartheta} e^{-\epsilon t}\left\|v_{10}\right\|_{q} \leq\left\|v_{10}\right\|_{\infty} \quad \text { for all } t \in\left(\tau, T_{\max }\right),
$$

where $\vartheta \in\left(\frac{n}{2 q}, 1\right), q>n$ and $\epsilon>0$. 
For $E_{2}$, set $m=0, q>n$ and $p=\infty$ in Lemma 2.2, so we can choose $\theta \in\left(\frac{n}{2 q}, \frac{1}{2}\right)$. In this case, we have $\varepsilon \in\left(0, \frac{1}{2}-\theta\right)$. Then there exist positive constants $C_{10}$ and $\mu$ such that

$$
\begin{aligned}
\left\|E_{2}(\cdot, t)\right\|_{\infty} & \leq C_{2}\left\|\left(A_{d_{2}}+1\right)^{\theta} E_{2}(\cdot, t)\right\|_{q} \\
& =\chi_{1} C_{2} \int_{0}^{t}\left\|\left(A_{d_{2}}+1\right)^{\theta} e^{-(t-s)\left(A_{d_{2}}+1\right)} \nabla \cdot\left(S\left(v_{1}(\cdot, t)\right) \nabla u(\cdot, t)\right)\right\|_{q} d s \\
& \leq \chi C_{4} \int_{0}^{t} e^{-(t-s)}\left\|\left(A_{d_{2}}+1\right)^{\theta} e^{-(t-s) A_{d_{2}}} \nabla \cdot\left(S\left(v_{1}(\cdot, t)\right) \nabla u(\cdot, t)\right)\right\|_{q} d s \\
& \leq C_{10} \int_{0}^{t}(t-s)^{-\theta-\frac{1}{2}-\varepsilon} e^{-(\mu+1)(t-s)}\left\|S\left(v_{1}(\cdot, t)\right) \nabla u(\cdot, t)\right\|_{q} d s
\end{aligned}
$$

for all $t \in\left(0, T_{\max }\right)$. From (3.9), we have

$$
\|\nabla u(\cdot, t)\|_{\infty} \leq H(\tau) \quad \text { for all } t \in\left(\tau, T_{\max }\right)
$$

Hence, there exists $C_{11}>0$ such that

$$
\left\|S\left(v_{1}(\cdot, t)\right) \nabla u(\cdot, t)\right\|_{q} \leq C_{11} \quad \text { for all } t \in\left(\tau, T_{\max }\right)
$$

Therefore, we obtain, for all $t \in\left(\tau, T_{\max }\right)$,

$$
\begin{aligned}
\left\|E_{2}(\cdot, t)\right\|_{\infty} & \leq C_{11} C_{12} \int_{0}^{t}(t-s)^{-\theta-\frac{1}{2}-\varepsilon} e^{-(\mu+1)(t-s)} d s \\
& \leq C_{11} C_{12} \int_{0}^{\infty} \sigma^{-\theta-\frac{1}{2}-\varepsilon} e^{-(\mu+1) \sigma} d \sigma \\
& \leq C_{13} \Gamma\left(\frac{1}{2}-\theta-\varepsilon\right)
\end{aligned}
$$

where $\Gamma(x)$ is the Gamma function. Since $\frac{1}{2}-\theta-\varepsilon>0$, then $\Gamma\left(\frac{1}{2}-\theta-\varepsilon\right)$ is positive and real-valued.

Finally, for $E_{3}$, by using (2.4) and (2.5), we have

$$
\begin{aligned}
\left\|E_{3}(\cdot, t)\right\|_{1, p} & \leq C_{2}\left\|\left(A_{d_{2}}+1\right)^{\theta} E_{3}(\cdot, t)\right\|_{q} \\
& \leq C_{3} \int_{0}^{t}(t-s)^{-\theta} e^{-\gamma(t-s)}\left\|v_{1}\left(f_{1}(u)-v_{1}-v_{2}\right)\right\|_{q} d s \\
& \leq C_{3} \int_{0}^{t}(t-s)^{-\theta} e^{-\gamma(t-s)}\left\|v_{1} f_{1}(u)\right\|_{q} d s \\
& \leq C_{3} \int_{0}^{t}(t-s)^{-\theta} e^{-\gamma(t-s)}\left(\left\|v_{1}(\cdot, t)\right\|_{q}+E\|u(\cdot, t)\|_{q}\right) d s \\
& \leq C_{3} \int_{0}^{t}(t-s)^{-\theta} e^{-\gamma(t-s)} d s \\
& \leq C_{3} \int_{0}^{\infty} \sigma^{-\theta} e^{-\gamma \sigma} d \sigma \leq C_{3} \Gamma(1-\theta) \quad \text { for all } t \in\left(\tau, T_{\max }\right)
\end{aligned}
$$


where $C_{3}$ denotes a generic constant that may vary from line to line, and $\Gamma(1-\theta)>0$ for $1-\theta>0$. For $p>n$, from the Sobolev embedding theorem, we have

$$
\left\|E_{3}(\cdot, t)\right\|_{\infty} \leq C_{14} \Gamma(1-\theta) \quad \text { for all } t \in\left(\tau, T_{\max }\right) .
$$

Therefore, by (3.22), (3.26) and (3.28), we see that $\left\|v_{1}(\cdot, t)\right\|_{\infty}$ is bounded for $t \in\left(0, T_{\max }\right)$.

Similarly, we see that $\left\|v_{2}(\cdot, t)\right\|_{\infty}$ is bounded for $t \in\left(0, T_{\max }\right)$.

Lemma 2.1 part 2 implies that $T_{\max }=\infty$ and therefore $\left(u(x, t), v_{1}(x, t), v_{2}(x, t)\right)$ is bounded for all $(x, t) \in \Omega \times(0, \infty)$.

Theorem 3.7 Let $\Omega$ be a bounded domain in $\mathbb{R}^{n}(n \geq 1)$ with smooth boundary $\partial \Omega$. Suppose that $d_{1}, d_{2}, d_{3}>0, \chi_{1} \geq 0, \chi_{2} \geq 0,(F),\left(H_{1}\right)$ and $\left(H_{2}\right)$ hold. For any $\left(u_{0}, v_{10}, v_{20}\right) \in$ $\left[W^{1, p}(\Omega)\right]^{3}$ where $p>n$, satisfying $u_{0}(x) \geq 0, v_{10}(x) \geq 0, v_{20}(x) \geq 0$ for $x \in \Omega$, the system $(1.1)$ possesses a unique global classical solution $\left(u(x, t), v_{1}(x, t), v_{2}(x, t)\right)$ satisfying $\left(u, v_{1}, v_{2}\right) \in$ $\left(C\left([0, \infty) ; W^{1, p}(\Omega)\right) \cap C^{2,1}(\bar{\Omega} \times(0, \infty))\right)^{3}$, and $\left(u(x, t), v_{1}(x, t), v_{2}(x, t)\right)$ is uniformly bounded in $\Omega \times(0, \infty)$, i.e. there is a constant $M\left(u_{0}, v_{10}, v_{20}\right)>0$ such that $\|u(\cdot, t)\|_{\infty}+\left\|v_{1}(\cdot, t)\right\|_{\infty}+$ $\left\|v_{2}(\cdot, t)\right\|_{\infty} \leq M\left(u_{0}, v_{10}, v_{20}\right)$ for all $t \in[0, \infty)$.

Proof Combining the results established in Lemma 2.1 and Theorem 3.6, we obtain the desired conclusions.

\section{Conclusions}

This paper focuses on the global existence and boundedness of system (1.1) under more general conditions. The fact that two predators compete for one prey species makes it harder to study the global dynamics for this model, and our analysis would also apply to other three component systems for the $L^{\infty}$ estimates.

\section{Acknowledgements}

The authors would like to thank the anonymous referees for their good comments. This work is supported by Natural Science Foundation of of Heilongjiang Province of China (No.A 2017007) and the Ph.D. Programs Foundation of Harbin University of China (No. HUDF 2016-001).

\section{Competing interests}

We claim that none of the authors have any competing interests in the manuscript.

\section{Authors' contributions}

The authors contributed equally to this paper. All authors read and approved the final manuscript.

\section{Publisher's Note}

Springer Nature remains neutral with regard to jurisdictional claims in published maps and institutional affiliations.

Received: 28 November 2017 Accepted: 8 March 2018 Published online: 27 March 2018

\section{References}

1. Kazarinov, N., van den Driessche, P.: A model predator-prey systems with functional response. Math. Biosci. 39, 125-134 (1978)

2. Lotka, A.J.: Elements of Physical Biology. Williams \& Wilkins, Baltimore (1925)

3. Medvinsky, A.B., Petrovskii, S.V., Tikhonova, I.A., Malchow, H., Li, B.L.: Spatiotemporal complexity of plankton and fish dynamics. SIAM Rev. 44(3), 311-370 (2002)

4. Murray, J.D.: Mathematical Biology. I. An Introduction. 3rd edn. Interdisciplinary Applied Mathematics, vol. 17. Springer, New York (2002)

5. Owen, M.R., Lewis, M.A.: How predation can slow, stop or reverse a prey invasion. Bull. Math. Biol. 63, 655-684 (2001)

6. Rosenzweig, L.M.: Paradox of enrichment: destabilization of exploitation ecosystems in ecological time. Science 171(3969), 385-387 (1971)

7. Meixner, M., De Wit, A., Bose, S., Schöll, E.: Generic spatiotemporal dynamics near codimension-two Turing-Hopf bifurcations. Phys. Rev. E 55, 6690-6697 (1997) 
8. Ricard, M.R., Mischler, S.: Turing instabilities at Hopf bifurcation. J. Nonlinear Sci. 19, 467-496 (2009)

9. Ko, W., Ryu, K.: Qualitative analysis of a predator-prey model with Holling type II functional response incorporating a prey refuge. J. Differ. Equ. 231, 534-550 (2006)

10. Wang, J.F., Shi, J.P., Wei, J.J.: Dynamics and pattern formation in a diffusive predator-prey system with strong Allee effect in prey. J. Differ. Equ. 251, 1276-1304 (2011)

11. Wang, J.F., Shi, J.P., Wei, J.J.: Global bifurcation analysis and pattern formation inhomogeneous diffusive predator-prey systems. J. Differ. Equ. 260, 3495-3523 (2016)

12. Wu, S.N., Shi, J.P., Wu, B.Y.: Global existence of solutions and uniform persistence of a diffusive predator-prey model with prey-taxis. J. Differ. Equ. 260(7), 5847-5874 (2016)

13. Winkler, M.: Absence of collapse in a parabolic chemotaxis system with signal-dependent sensitivity. Math. Nachr. 283, 1664-1673 (2010)

14. Amann, H.: Dynamic theory of quasilinear parabolic equations II, Reaction-diffusion systems. Differ. Integral Equ. 3, $13-75$ (1990)

15. Amann, H.: Nonhomogeneous linear and quasilinear elliptic and parabolic boundary value problems. In: Function Spaces, Differential Operators and Nonlinear Analysis (Friedrichroda, 1992), Teubner-Texte Math., vol. 133, pp. 9-126 Teubner, Stuttgart (1993)

16. Horstmann, D., Winkler, M.: Boundedness vs. blow-up in a chemotaxis system. J. Differ. Equ. 215, $52-107$ (2005)

17. Nirenberg, L.: On elliptic partial differential equations. Ann. Sc. Norm. Super. Pisa 13(3), 115-162 (1959)

18. Winkler, M., Djie, K.C.: Boundedness and finite-time collapse in a chemotaxis system with volume-filling effect. Nonlinear Anal. 72, 1044-1064 (2010)

\section{Submit your manuscript to a SpringerOpen ${ }^{\circ}$ journal and benefit from:}

- Convenient online submission

- Rigorous peer review

- Open access: articles freely available online

- High visibility within the field

- Retaining the copyright to your article

Submit your next manuscript at $\boldsymbol{\wedge}$ springeropen.com 